\title{
Utilizing Half Convolutional Autoencoder to Generate User and Item Vectors for Initialization in Matrix Factorization
}

\author{
Tan Nghia Duong ${ }^{*}+{ }^{+}$, Nguyen Nam Doan ${ }^{+} \mathbb{C}$, Truong Giang Do ${ }^{+} \mathbb{(}$, , Manh Hoang Tran, Duc Minh Nguyen \\ and Quang Hieu Dang
}

check for updates

Citation: Duong, T.N.; Doan, N.N.; Do, T.G.; Tran, M.H.; Nguyen, D.M.; Dang, Q.H. Utilizing HCAE to Generate User and Item Vectors for Initialization in MF. Future Internet 2022, 14, 20. https://doi.org/ $10.3390 /$ fi 14010020

Academic Editor: Paolo Bellavista

Received: 3 November 2021

Accepted: 27 December 2021

Published: 4 January 2022

Publisher's Note: MDPI stays neutral with regard to jurisdictional claims in published maps and institutional affiliations.

Copyright: (c) 2022 by the authors. Licensee MDPI, Basel, Switzerland. This article is an open access article distributed under the terms and conditions of the Creative Commons Attribution (CC BY) license (https:// creativecommons.org/licenses/by/ $4.0 /)$.

\author{
School of Electronics and Telecommunications, Hanoi University of Science and Technology, \\ Hanoi 100000, Vietnam; nam.dn168746@sis.hust.edu.vn (N.N.D.); giang.dt172524@sis.hust.edu.vn (T.G.D.); \\ hoang.tranmanh@hust.edu.vn (M.H.T.); minh.nguyenduc1@hust.edu.vn (D.M.N.); \\ hieu.dangquang@hust.edu.vn (Q.H.D.) \\ * Correspondence: nghia.duongtan@hust.edu.vn \\ t These authors contributed equally to this work.
}

\begin{abstract}
Recommendation systems based on convolutional neural network (CNN) have attracted great attention due to their effectiveness in processing unstructured data such as images or audio. However, a huge amount of raw data produced by data crawling and digital transformation is structured, which makes it difficult to utilize the advantages of CNN. This paper introduces a novel autoencoder, named Half Convolutional Autoencoder, which adopts convolutional layers to discover the high-order correlation between structured features in the form of Tag Genome, the side information associated with each movie in the MovieLens $20 \mathrm{M}$ dataset, in order to generate a robust feature vector. Subsequently, these new movie representations, along with the introduction of users' characteristics generated via Tag Genome and their past transactions, are applied into well-known matrix factorization models to resolve the initialization problem and enhance the predicting results. This method not only outperforms traditional matrix factorization techniques by at least $5.35 \%$ in terms of accuracy but also stabilizes the training process and guarantees faster convergence.
\end{abstract}

Keywords: autoencoder; collaborative filtering; convolutional neural network; matrix factorization; recommendation system

\section{Introduction}

Information explosion has been occurring in the past decades thanks to the Internet. In social media, digital advertising, and especially e-commerce, this explosion not only makes people overwhelmed with a variety of choices but also challenges suppliers to retain their customers and compete with others by offering the most relevant items. As a result, Recommendation Systems (RSs), which provide automated and personalized recommendations to users, are critical.

In general, RSs have three main approaches [1]: content-based method, collaborative filtering method and hybrid method. Content-based methods [2,3] suggest items based on the contents of products and on users' preferences, which requires a substantial amount of item profiles and users' past behaviors. Consequently, the main disadvantage of content-based models is the lack of available and reliable product properties. In contrast, Collaborative Filtering $(\mathrm{CF})$ approaches $[4,5]$ do not require product information but rely on the analogy between users with similar tastes determined by their past transactions. There are two branches of the CF approach: memory-based (or neighborhood-based) and model-based. The memory-based branch focuses on computing the correlation between items or between users. Nevertheless, the existence of sparse rating matrices in practice significantly degrades the performance of these approaches. This is a common problem because customers are often not willing to rate items. On the other hand, the model-based 
(or latent factor) branch has shown its effectiveness on extremely sparse interaction matrices. The main idea is to project each user and item into a lower dimension space, then analyze the user-item interaction by dot product [6-8]. Several studies have shown that an appropriate initialization setting for matrix factorization can improve the speed and accuracy of matrix factorization models [9-11].

However, there are usually not enough transaction data to make accurate recommendations for a new user or item, which raises the cold-start problem in CF techniques. Therefore, hybrid methods are proposed to tackle this problem by combining both content-based and CF models [12,13]. Generally, hybrid methods integrate user-item ratings and auxiliary information to generate unified systems. As in [12], user profiles, movie genres and their past interactions are integrated into one model to predict dyadic response in a generalized linear framework. Barragáns et al. [13] proposed a hybrid approach that utilizes Singular Value Decomposition (SVD) to make television program recommendations to deal with the limitations of content-based and CF systems. One restriction of these works is the privacy of user profiles, which limits the shared personal information. In another work [7], after proving the advantage of SVD++ model, an integrated model between neighborhood-based and SVD++ is established to gain a better result by generating new representation for a user from the items rated by that user instead of using an explicit parameterization. A model named Factorization Machines (FM) combining matrix factorization and Support Vector Machine also uses both ratings and auxiliary information for predictions [14].

Studies on hybrid approaches for initialization problem in matrix factorization models have gained some attention recently. Hidasi and Tikk [15] used the similarity between users and items to initialize feature vectors by taking advantage of available contextual information. Additionally, Zhao et al. [16] adopted the item's attributes information to initialize the item feature matrix in SVD++ model; however, this method just accounted for only initializing item features and the overall improvement is modest. The common point of these methods is that features such as movie genres are considered as good representations of items. Nonetheless, in practice, raw content-based information needs to be preprocessed and extracted carefully to fit into a specific RS, especially when the contents of products are texts, images or videos which are hard to exploit semantic and meaningful representations.

While traditional methods have yielded promising results, the performances are still restricted by theirs linearity. For real-world data structure, deep learning can be a powerful approach to boost RSs owing to its outstanding capability to explore non-linear correlations between data features $[17,18]$. Among a variety of deep learning approaches, CNN provides an efficient neural network and is considered as an excellent feature extractor [19]. To find the correlation between user and songs, a music RS [20] was proposed where CNN was used to extract song's latent features from the audio data. Additionally, CNN was trained to transform item description documents into an embedding space, which later can be incorporated into CF models [21].

In the interest of CNN and its advantages, this paper concentrates on applying 1D$\mathrm{CNN}$ to enhance the ability to extract information of a classic autoencoder and provide scrupulous features to RSs. The result is followed by a novel method of integrating contentbased information to matrix factorization models. Our empirical studies are conducted on the MovieLens $20 \mathrm{M}$ dataset released in October 2016 [22]. Although the dataset includes a current copy of a movie Tag Genome based on user-contributed information which is often considered as content-related information, there are no statistics about user profiles. To deal with the challenges mentioned above, in addition to movies' auxiliary information, we incorporate the parameterized user features into the initialization of matrix factorization techniques. The main contributions of this paper are summarized as follows.

- $\quad$ Designing a CNN-based autoencoder named Half Convolutional Autoencoder (HCAE) where convolutional layers are used as a feature extractor to generate a lower dimensional descriptor for each movie regardless of the arrangement or the semantic relationships of original features, which helps to increase the accuracy of RSs. 
- Utilizing the content-based information produced by HCAE and available ratings to parameterize user preferences for resolving the initialization problem of modelbased method, which considerably improves the performance of the traditional matrix factorization models.

The remaining of the paper is organized as follows. Section 2 presents the formalized problem and discusses existing solutions. Our previous works and experimental settings are summarized in Sections 3 and 4, respectively. The proposed models and their performance are described in Sections 5 and 6 along with the state-of-the-art models for comparison. Finally, we conclude with a summary of this work in Section 7.

\section{Preliminaries}

In this paper, $u, v$ denote users and $i, j$ denote items. The rating that user $u$ gives item $i$ is denoted by $r_{u i}$ where higher values indicate stronger preference. $U_{i}$ is the set of all users that rate item $i$, and $U_{i j}$ is the set of all users that rate both items $i$ and $j$ whilst $R(u)$ denotes the set of all items rated by user $u$.

Two popular $\mathrm{CF}$ techniques and three common autoencoder architectures are briefly introduced as follows.

\subsection{Memory-Based CF}

Based on the similarity between users or items, the memory-based CF technique tries to predict the most appropriate recommendations to users. There are two forms of memory-based CF: (i) user-oriented (or user-user) CF [23] and (ii) item-oriented (or item-item) CF [24]. Of the two approaches, the latter is more favored in practice due to its superior accuracy and better scalability [5]. An item-item CF system (ii-CF) recommends to a specific user those items which are the most relevant to the items rated or purchased by her.

At the core of these systems is a similarity measure which indicates the analogy $s_{i j}$ between two items. After computing the similarity degree between items using popular similarity measures such as Cosine similarity function (Cos) or Pearson Correlation Coefficients (PCC), the $k$ most similar items of item $i$ rated by user $u$ can be identified. This set of $k$ items is called k-nearest neighbors $(\mathrm{kNN})$ which is denoted by $S^{k}(i, u)$. The most simple formula to estimate $\hat{r}_{u i}$ is a weighted average of the ratings of similar items $i \in S^{k}(i, u)$ (named kNNBasic model):

$$
\hat{r}_{u i}^{k N N B a s i c}=\frac{\sum_{j \in S^{k}(i, u)} s_{i j} r_{u j}}{\sum_{j \in S^{k}(i, u)} s_{i j}}
$$

A modification of Equation (1) adjusts the final prediction using baseline estimate, hence the name kNNBaseline model [25], as follows:

$$
\hat{r}_{u i}^{k N N B a s e l i n e}=b_{u i}+\frac{\sum_{j \in S^{k}(i ; u)} s_{i j}\left(r_{u j}-b_{u j}\right)}{\sum_{j \in S^{k}(i, u)} s_{i j}}
$$

Besides Cos and PCC, advanced similarity measures, such as PCCBaseline [25] or cubedPCC [26], also effectively improve the performance of kNN models.

\subsection{Model-Based CF}

Among various model-based CF techniques, latent factor models are the most popular because they can overcome the weakness of neighborhood-based approaches on extremely sparse data. This type of model aims at uncovering latent features that explain the observed ratings, as proven in Netflix Prize competition [6].

Let $R \in \mathbb{R}^{m \times n}$ denote the rating matrix where $m$ is the number of users, $n$ is the number of items. By applying SVD factorization, both users and items are mapped into a latent space of dimension $k(k \ll m, n)$. Here, each user can be characterized by a user-factor 
vector $p_{u} \in \mathbb{R}^{k}$, and each item by an item-factor vector $q_{i} \in \mathbb{R}^{k}$. The prediction is estimated by taking the following inner product.

$$
\hat{r}_{u i}=q_{i}^{T} p_{u}
$$

Rating value estimated in Equation (3) raises a bias problem in practice. Among users with similar interests, some users tend to give higher ratings than others. Among similar items, some always get lower ratings than others due to irrelevant reasons such as poor video quality. Therefore, user and item-specific biases, $b_{u} \in \mathbb{R}^{m}$ and $b_{i} \in \mathbb{R}^{n}$, respectively, are introduced. Specifically, the rating user $u$ give to the item $i$ is approximated as

$$
\hat{r}_{u i}=b_{u i}+q_{i}^{T} p_{u}
$$

where $b_{u i}=\mu+b_{u}+b_{i}$ is the baseline estimate rating of user $u$ for item $i$, and $\mu$ is the mean of ratings.

Non-negative Matrix Factorization (NMF) is another common model in the study of RS [27-29]. Different from regular matrix factorization, the objective of NMF is to factorize a non-negative matrix into matrices with no negative element. This constraint is useful in many fields such as computer vision, signal processing or RS in general where non-negative data are explicitly required.

Even though the idea of mapping the interaction matrix into a lower-dimension latent space provides remarkable accuracy, the latent factors themselves have no explicit meaning. The predicted ratings, therefore, are unpersuasive and untrustworthy, which declines user satisfaction of RSs [30]. This problem also makes it challenging for engineers and researchers to evaluate, diagnose and refine the system in the long term.

\subsection{Autoencoder}

Among current techniques for dimensionality reduction and information retrieval, an autoencoder (AE) is widely used not only as a nonlinear decomposition to replace traditional linear inner product but also as a representation learning mechanism [31]. It eliminates the information redundancy by mapping data from high feature space to lower one, generating more precise and efficient data representation.

Figure 1 illustrates the structure of a simple feedforward 1-layer AE. Generally, an AE is constituted by two main parts:

- An encoder $\phi: \mathcal{X} \rightarrow \mathcal{C}$ that maps the input features $\mathcal{X}$ into the code.

- A decoder $\psi: \mathcal{C} \rightarrow \mathcal{X}$ that reconstructs the original features from the code.

The code $\mathcal{C}$ itself is the hidden layer output that is usually used to characterize the input. The objective of $\mathrm{AE}$ is to minimize the difference between the input and the output by reconstructing $\mathcal{X}$ from the reduced encoding $\mathcal{C}$ as follows:

$$
\begin{aligned}
\phi, \psi & =\underset{\phi, \psi}{\arg \min }\|\mathcal{X}-(\psi \circ \phi) \mathcal{X}\|^{2} \\
& =\underset{\phi, \psi}{\arg \min } \| \mathcal{X}-\left(\psi(\phi(\mathcal{X})) \|^{2}\right.
\end{aligned}
$$

In practice, only the code $\mathcal{C}$ is extracted to create a compressed representation of the input data that preserves the most crucial information for further analysis.

Several modifications on the AE architecture have been introduced recently to improve the effectiveness of representation learning and dimensionality reduction. To prevent the autoencoder from learning the identity function, Denoising Autoencoder (DAE) first corrupts the input by adding noise, then learns to predict the initial data point as the output [32]. Meanwhile, despite sharing a similar architecture with a vanilla AE, Variational Autoencoder (VAE) [33] encodes the input into a multivariate latent distribution rather than a vector. 
There have been several studies successfully applying AE and its variants into RS. Collaborative Deep Learning (CDL) [34] was proposed for joint learning side information of items by a Stacked Denoising Autoencoder. Additionally, an AE-based model for rating prediction is the item-based AutoRec (I-AutoRec) [35] which fills missing ratings by applying an AE to reconstruct its input containing known ratings. In contrary to CDL, I-AutoRec directly handles explicit data to make reliable predictions without the side information. Other variations of AE [36,37] can also optimize the network input, capturing high-order latent factors from users and items.

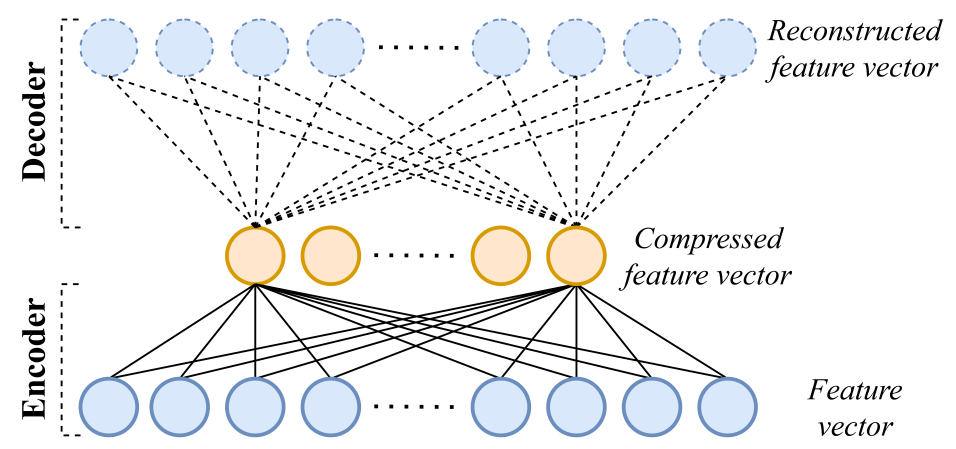

Figure 1. Illustration of a vanilla 1-layer AE. The input and output layers have the same number of neurons while the hidden layer is smaller to serve the idea of mapping original data from a high dimension space into a lower one.

\section{Previous Work}

In [38], a number of problems regarding similarity measurement method using the rating information were noticed. Firstly, the rating matrix in practice is extremely sparse (for example, $99.47 \%$ entries of this matrix in the MovieLens $20 \mathrm{M}$ dataset are missing), which makes it hard to evaluate the relevance between two items that have many ratings but only share a few common users. Secondly, calculating the similarity score between two items is a time-consuming task due to a large number of users (usually in the order of millions). To address these problems, a novel similarity measure was proposed using Genome Tags instead of rating information. Specifically, each movie is characterized by a genome score vector $g=\left\{g_{1}, g_{2}, \ldots, g_{1128}\right\}$ which encodes how strong a movie exhibits particular properties represented by 1128 tags [22], and the similarity score $s_{i j}$ between movies $i$ and $j$ is calculated as follows.

$$
s_{g_{i}, g_{j}}^{\text {Cosgenome }}=\frac{\sum_{k=1}^{G} g_{i k} \times g_{j k}}{\sqrt{\sum_{k=1}^{G} g_{i k}^{2}} \times \sqrt{\sum_{k=1}^{G} g_{j k}^{2}}}
$$

or

$$
s_{g_{i}, g_{j}}^{P C C^{\text {genome }}}=\frac{\sum_{k=1}^{G}\left(g_{i k}-\bar{g}_{i}\right) \times\left(g_{j k}-\bar{g}_{j}\right)}{\sqrt{\sum_{k=1}^{G}\left(g_{i k}-\bar{g}_{i}\right)^{2}} \times \sqrt{\sum_{k=1}^{G}\left(g_{j k}-\bar{g}_{j}\right)^{2}}}
$$

where $\bar{g}_{i}$ and $\bar{g}_{j}$ are the mean genome scores of vectors $g_{i}$ and $g_{j}$, respectively; and $G=1128$ is the length of genome vectors. Experiments conducted on the preprocessed MovieLens $20 \mathrm{M}$ dataset (keeping only movies with Tag Genome) showed that the item-oriented CF models based on similarity measures $\mathrm{Cos}_{\text {genome }}$ and $\mathrm{PCC}_{\text {genome }}$ provide accuracy equivalent to the state-of-the-art CF models using rating information whilst performing at least 2 times faster.

In [39], we introduced a natural language processing (NLP)-based cleaning process to eliminate the redundancy from the original 1128 genome tags to generate a more accurate description for each movie with 1044 new tags. While this process slightly improved the accuracy of the systems, the number of new tags is still quite large (7\% smaller to original 1128 tags) and other groups of related tags which cannot be combined using NLP become 
hidden. Hence, a 3-layer AE was implemented to compress cleaned tags into a vector of 600 elements in order to discover the latent characteristics inside the genome tags and regenerate a more powerful representation of each movie. By using new feature vectors for every movie, kNN-Content ${ }^{\mathrm{AE}}$ model can achieve at least $2.56 \%$ lower RMSE when compared to its counterparts while the prediction time is still reasonable.

Additionally, a novel technique integrating the matrix factorization output as the baseline estimate of user rating into kNNBaseline model was described as follows.

$$
\hat{r}_{u i}^{k N N-\text { Content }}=\hat{r}_{u i}+\frac{\sum_{j \in S^{k}(i ; u)} s_{i j}\left(r_{u j}-\hat{r}_{u j}\right)}{\sum_{j \in S^{k}(i ; u)} s_{i j}}
$$

where $\hat{r}_{u i}$ and $\hat{r}_{u j}$ are the predicted ratings produced by SVD/SVD++ model while $s_{i j}$ is calculated by kNN-Content ${ }^{\mathrm{AE}}$ model. This created hybrid content-based and CF models, kNN-Content ${ }^{\mathrm{AE}}$-SVD and kNN-Content ${ }^{\mathrm{AE}}$-SVD++, which take the advantages of both local level interaction of neighborhood-based method and global level characteristics explored by model-based method to provide more precise recommendations.

\section{Experimental Setup}

\subsection{Dataset}

In the literature, the two most popular datasets in recommendation system are the MovieLens dataset [22] and the Netflix Prize dataset [40]. While the former is continuously updated and complimented with new auxiliary knowledge for movies, the latter not only focuses on rating information associated with limited secondary data (only including movie's title and year of release, and rating dates), but it has not been revised since the competition started due to privacy concerns. Therefore, in order to evaluate the performance of the proposed models, the MovieLens $20 \mathrm{M}$ dataset is chosen as a benchmark in this work.

Released by GroupLens in 2015, this dataset originally contains 20,000,263 ratings and is updated in 2016 with the latest 465,564 tag applications across 27,278 movies created by 138,493 users (all selected users had rated at least 20 movies). The ratings range from 0.5 to 5.0 with a step of 0.5 . Tag Genome data encodes how strongly movies exhibit particular properties represented by tags in the range of 0 to 1 which is computed using user-contributed content including tags, ratings, and textual reviews [22].

It is necessary to preprocess the original dataset. Any movie which does not have Tag Genome is discarded from the dataset. Only movies and users containing at least 20 ratings are kept. Table 1 summarizes the results: eventually, the preprocessed dataset consists of $19,793,342$ ratings (approximately $98.97 \%$ sparsity compared to $99.47 \%$ sparsity of the original dataset) given by 138,185 users for 10,239 movies.

Table 1. Summary of the original MovieLens $20 \mathrm{M}$ and the preprocessed dataset.

\begin{tabular}{lcccc}
\hline & \# Ratings & \# Users & \# Movies & Sparsity \\
\hline Original dataset & $20,000,263$ & 138,493 & 27,278 & $99.47 \%$ \\
\hline Preprocessed dataset & $19,793,342$ & 138,185 & 10,239 & $98.97 \%$ \\
\hline
\end{tabular}

\subsection{Evaluation Scheme}

The preprocessed dataset is split into 2 distinct parts: $80 \%$ ratings of each movie are used as the training set, and the $20 \%$ remaining as the testing set. To compare the performance between models, the following widely-used indicators are used: 
- $\quad$ RMSE (Root Mean Squared Error) for rating prediction task: evaluate the errors of the predicted ratings where smaller values provide more accurate recommendations:

$$
\text { RMSE }=\sqrt{\sum_{u, i \in \mathrm{TESTSET}}\left(\hat{r}_{u i}-r_{u i}\right)^{2} /|\mathrm{TESTSET}|}
$$

where $\mid$ TESTSET $\mid$ is the size of the testing set, $\hat{r}_{u i}$ denotes the predicted rating of user $u$ to item $i$ estimated by the model, and the corresponding observed rating in the testing set is denoted by $r_{u i}$.

- $\quad$ Precision@k (P@k) and Recall@k (R@k) for the ranking (top-k recommendation) task: Precision@ $k$ is defined as a fraction of relevant items among the top $k$ recommended items, and Recall@ $k$ is defined as a fraction of relevant items in the top $k$ recommended items among all relevant items. Higher $\mathrm{P} @ k$ and $\mathrm{R} @ k$ indicate that more relevant items are recommended to users.

- Time [s] for timing evaluation: the total duration of the model's learning process on the training set and predicting all samples in the testing set.

All experiments in this work are conducted on a workstation consisting of an Intel Xeon Processor E5-2637 v3 3.50 GHz (2 processors) with 32 GB RAM and no GPU.

\subsection{Baselines and Experimental Settings}

To evaluate the performance of proposed models, the following baseline models are implemented.

- $\quad$ ii-CF [24]: the similarity score between movies is measured using PCCBaseline with the number of neighbors is set at 40 .

- $\quad$ SVD [6] and SVD++ [7]: both models are trained using 40 hidden factors with 100 iterations and the step size of 0.002 .

- NMF: optimization procedure is a regularized SGD based on Euclidean distance error function [41] with regularization strength of 0.02 and 40 hidden factors.

- $\quad$ kNN-Content [38]: PCC genome is used as the similarity measure.

- $\quad$ kNN-Content ${ }^{\mathrm{AE}}$-SVD and kNN-Content ${ }^{\mathrm{AE}}$-SVD++ [39]: 600-feature vectors for movies are learned from 1044 NLP-preprocessed genome tags using a 3-layer AE.

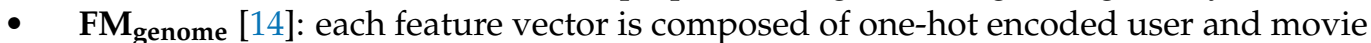
ID, movie genres and genome scores associated with each movie; the model is trained with degree $d=2$ and 50 iterations.

- I-AutoRec [35]: a 3-layer AE is trained using 600 hidden neurons, and the combination of activation functions is (Identity, Sigmoid).

In our experiments, the optimal hyperparameters for each baseline method above are carefully selected using 5-fold cross validation to guarantee fair comparisons. For ii-CF model, the number of neighbors is chosen from $\{10,20,30,40,50,100,150\}$, and the similarity measures implemented are Cos, PCC and PCCBaseline. For SVD, SVD++, and NMF models, the number hidden factors is chosen from $\{20,30,40,50,60,80,100\}$. These traditional models are reimplemented using Numba compiler (https:/ / github.com/ numba/numba, accessed on 2 November 2021) to optimize their performances [42]. For I-AutoRec model, the size of the hidden layer is set for $n \in\{200,400,600,800,1000\}$ units; and the choices of activation functions $f(\cdot), g(\cdot)$ are experimented with (Identity, Identity), (Identity, Sigmoid), (Sigmoid, Identity), (Sigmoid, Sigmoid). Finally, the regularization strength is tuned $\lambda \in\{0.001,0.002,0.01,0.02,0.1,1,2\}$ for all baselines.

In this paper, we have two main contributions. In the following, a novel autoencoder named Half Convolutional Autoencoder is first introduced to capture the fundamental characteristics of each movie from its original Tag Genome. The ability to extract essential features of an HCAE is empirically proven to outperform that of the classical AE and its variants via the application in various RSs. Secondly, through the newly generated representation for each movie, a user's interest is vectorized by the movies she rated. 
Both the movie and user vectors are then adopted to initialize the latent vectors in matrix factorization models, which significantly boosts the precision of the systems.

\section{Half Convolutional Autoencoder}

\subsection{Learning New Representation of Structured Data with an HCAE}

Experimental results in our previous work [39] demonstrated the capability of an AE to produce a more compact and powerful representation for movies than the original genome scores. However, the fully-connected architecture of a traditional AE does not consider the order of its raw inputs, which takes a risk that the network just tries to learn the data without extracting any more useful information [19].

Compared to conventional fully connected neural networks, a typical CNN does not require a large number of neurons for high dimensional inputs, which enables the training process to be much more efficient with fewer parameters. Additionally, CNNs are widely recognized as a robust feature extractor that is commonly applied on unstructured data such as images, videos or audio where elements of a data point spatially and/or temporally close to each other share similar information, and their positioning can negatively affect the performance if arranged arbitrarily [43].

Meanwhile, data in common RSs are mostly stored in tabular form such as rating matrix, user profiles, or movie genres (for example, the MovieLens datasets). This kind of structured data has generally little or no spatial/temporal knowledge and is considered to be suitable for fully connected networks. Hence, to our knowledge, there is little effort in applying a CNN as the feature extractor in RSs despite its effectiveness.

As stated above, this paper focuses on the MovieLens $20 \mathrm{M}$ dataset which introduces a new type of information associated with each movie named Tag Genome. Investigating this kind of tabular data provides us some prospective reasons for applying a CNN in order to generate a more precise representation for each movie against a vanilla AE:

- $\quad$ Firstly, we reorganize Tag Genome data into a table so that each movie is represented as a row and its genome scores are stored in the columns (as in Table 2). It can be seen that each row has fully numerical values in the range of $[0,1]$ which indicate the strength of the relevance between a movie and its corresponding genome tags (i.e., attributes). If each row is assumed to be a discrete-time signal where the attributes' positions are treated as "timestamps" and their values as the displacements, each movie will be characterized by a signal bringing its information. Moreover, the attributes are generally independent of each other, so this signal resembles a random vibration illustrated in Figure 2 that we could apply a 1D-CNN to extract its features [44].

- Secondly, if the order of the columns is shuffled simultaneously for all movies, the physical shapes of the signals will change in a consistent way (i.e., the correlation between a random pair of signals remains unchanged) and still deliver the knowledge about the movies. In other words, the above assumption holds true regardless of the positioning of the movie attributes into the table. Consequently, a 1D-CNN still has the potential to perform feature extraction on the new signals.

We also tried to apply a 2D-CNN on these "vibration" signals using a proper 1D-to-2D conversion. A common technique is to reshape the signals into $m \times n$ matrices before feeding them into a 2D-CNN $[45,46]$. Nonetheless, this technique requires the length of the input signals to be a non-prime number and often costs a higher computational complexity than its 1D counterpart. Therefore, we opt to choose a 1D-CNN for our purpose. 
Table 2. Reorganizing original Tag Genome data into a tabular form. Each movie is presented as a row and its genome scores are stored in the columns.

\begin{tabular}{lcccccc}
\hline & Tag \#1 & Tag \#2 & Tag \#3 & $\ldots$ & Tag \#1127 & Tag \#1128 \\
\hline Toy Story (1995) & 0.0250 & 0.0250 & 0.0578 & $\ldots$ & 0.0778 & 0.0230 \\
GoldenEye (1995) & 0.9998 & 0.9998 & 0.0195 & $\ldots$ & 0.0730 & 0.0183 \\
Titanic (1997) & 0.0380 & 0.0345 & 0.1190 & $\ldots$ & 0.0693 & 0.0210 \\
Resident Evil (2002) & 0.0403 & 0.0350 & 0.0195 & $\ldots$ & 0.9588 & 0.9598 \\
White Zombie (1932) & 0.0188 & 0.0203 & 0.0270 & $\ldots$ & 0.9670 & 0.9840 \\
$\ldots$ & $\ldots$ & $\ldots$ & $\ldots$ & $\ldots$ & $\ldots$ & $\ldots$ \\
\hline
\end{tabular}

Toy Story (1995)

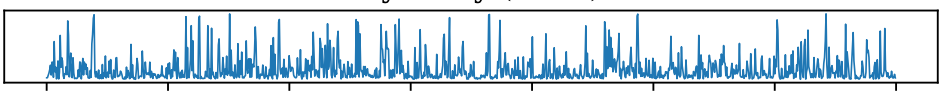

GoldenEye (1995)

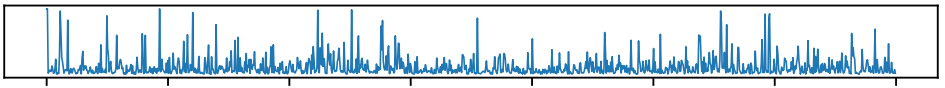

Titanic (1997)

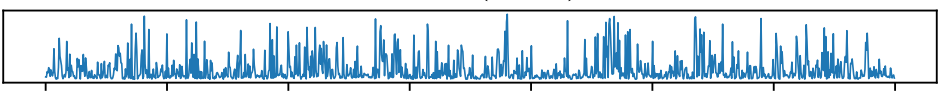

Resident Evil (2002)

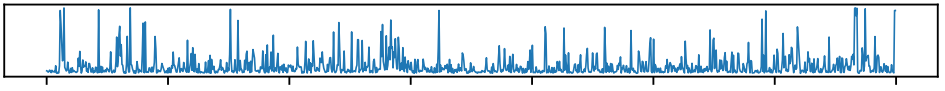

White Zombie (1932)

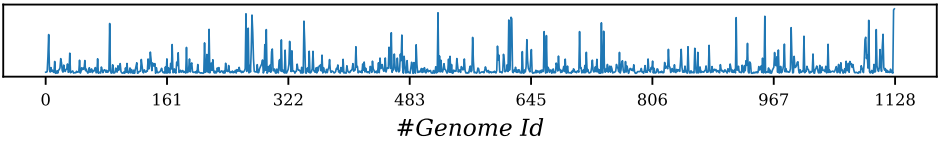

Figure 2. Random vibration signals of the 5 movies in MovieLens $20 \mathrm{M}$ dataset resembled by Tag Genome data.

In this work, a novel autoencoder architecture named Half Convolutional Autoencoder is devised to harness the capability of a 1D-CNN in order to explore the essential characteristics of the movies from the available Tag Genome data. The structure of an HCAE is illustrated in Figure 3. Specifically, a complete forward-propagation process of HCAE can be described in the following steps:

1. A feature vector representing a random vibration signal is fed to the input layer of the HCAE;

2. Each neuron of the 1D-Convolution layer performs a linear convolution between the signal and corresponding filter to generate the input feature map of the neuron;

3. The input feature map of each neuron is passed through the activation function to generate the output feature map of the neuron of the convolution neuron;

4. In the 1D-Pooling layer, each neuron's feature map is created by decimating the output feature map of the previous neuron of the 1D-Convolution layer to reduce the dimensions of the final feature maps;

5. In the Flatten layer, the output feature maps are flattened into a single feature vector, which is forward-propagated through the following fully-connected Compression layer to encode the feature map into lower dimensional space;

6. The decoder structure remains fully connected like a vanilla $\mathrm{AE}$, where the output code are forward-propagated through a fully connected decoder to reconstruct the original feature vector, or the 1D-signal.

Compared to a typical AE, the fundamental difference of the HCAE comes from the asymmetry of the Half Convolutional encoder and decoder parts, hence its name. 


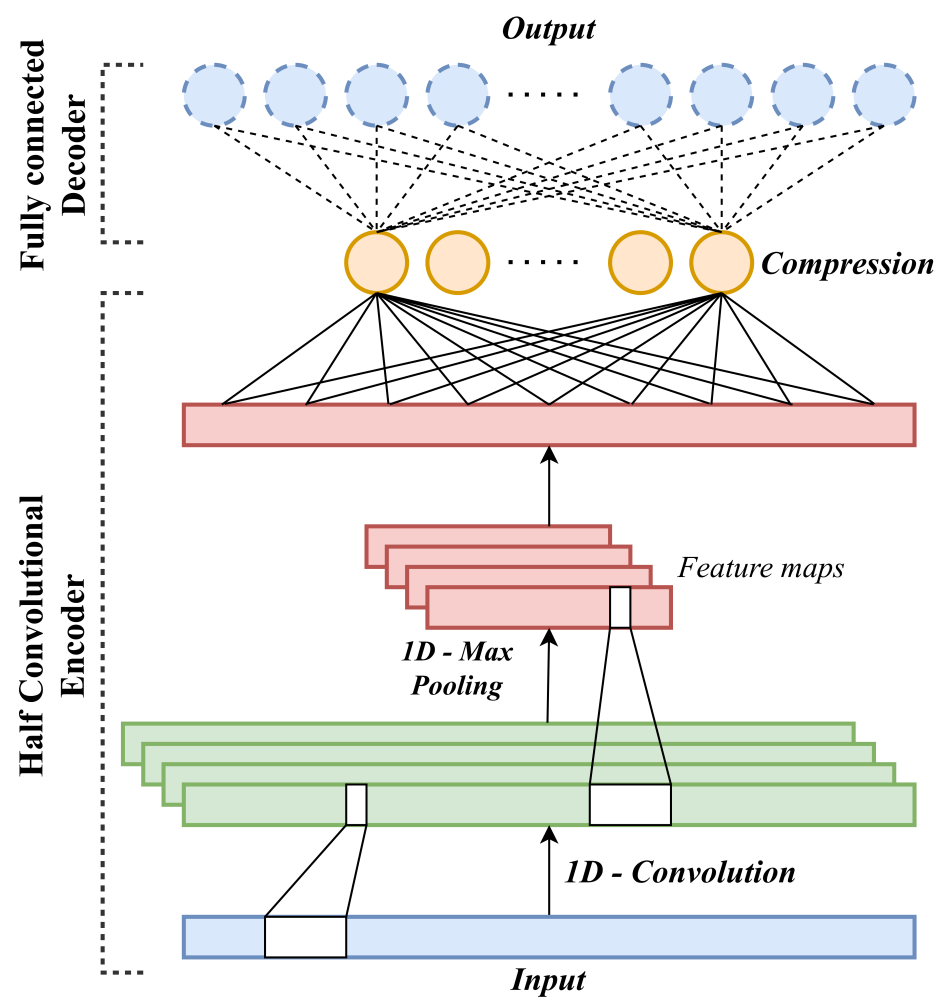

Figure 3. Architecture of the proposed HCAE: the encoder is based on a 1D-CNN whilst the decoder remains fully connected.

In more detail, the encoder part of the newly proposed architecture works as follows. For each filter $F_{i}$, the 1D-Convolution layer, along with the 1D-Max Pooling layer, learns the feature map $d_{i}=P\left(g\left(\mathcal{X} * F_{i}\right)\right)$ from input feature vector $\mathcal{X}$, where $g(*)$ is the activation function of the Convolution layer, and $P(*)$ denotes the Max Pooling transformation. These feature maps are then transformed to a $1 \mathrm{D}$-vector $\mathbf{d}$ via a Flatten layer before eventually being fed into a Compression layer to generate a compact representation of a movie. Here the output sizes of the Convolution layer $n_{\text {out }}$ with the padding size $p$ and of the Max Pooling layer $n_{\text {out }}^{\prime}$ are calculated using the following formulas:

$$
\begin{gathered}
n_{\text {out }}=\left(\frac{n_{\text {in }}+2 p-f}{s}\right)+1 \\
n_{\text {out }}^{\prime}=\left(\frac{n_{\text {in }}^{\prime}-f^{\prime}}{s^{\prime}}\right)+1
\end{gathered}
$$

where $n_{i n}, n_{i n}^{\prime}$ denote the input sizes; $f, f^{\prime}$ are the kernel sizes; and $s, s^{\prime}$ are the stride sizes of the Convolution and Max Pooling layers, respectively.

Although the encoder implements a CNN architecture, the decoder works similarly to the one of a conventional AE: it tries to reconstruct the original input through a fully connected layer. In addition to possessing the same objective function as the one of a vanilla $\mathrm{AE}$, this structure makes the proposed HCAE easily adapt to any shape of the input vector, which is infeasible if a 1D-Up Sampling layer is deployed at the decoder in the case of a "full" Convolutional AE. That is due to the fact that the output size of a 1D-Up Sampling layer is computed as:

$$
n_{\text {UpSampling_out }}=n_{\text {UpSampling_in }} * f_{\text {UpSampling }}
$$

where $n_{\text {UpSampling }}$ in and $f_{\text {UpSampling }}$ denote the input size and the factor size of the layer, respectively. It is implied that Equation (12) imposes certain constraints on the values of these 3 factors. For instance, $n_{\text {UpSampling_out }}$ must be a non-prime; otherwise, $n_{\text {UpSampling_in }}$ 
has to be equal to $n_{\text {UpSampling_out }}$ and $f_{\text {UpSampling }}$ equal to 1 , which is almost meaningless. It is worth noting that $n_{\text {UpSampling_in }}$ matches the length of the Max Pooling layer's output, and $n_{\text {UpSampling_out }}$ is exactly the original input size of the HCAE. Therefore, this causes restrictions on the input shape as well as the hyperparameter selection of the proposed architecture.

\subsection{Utilizing HCAE in Recommendation Systems}

In our experiments, the number of filters and the kernel size of the Convolution layer are firstly configured at 4 and 5, respectively. However, we later show that the overall performance of the HCAE is almost not affected by the values of these settings. The pooling size and the stride size are set equal to the number of filters so that the output of the Flatten layer has the same dimension as the HCAE input. Comprehensive experiments show that the dropout rate of 0.2 between the Compression and the Output layer is effective in preventing overfitting. The optimization algorithm used in this work is Adam due to little hyperparameter-tuning requirement, computational efficiency and faster convergence [47].

Activation of the decoder is Sigmoid function which returns the value between 0 and 1 to match the range of the genome scores. To find the optimal activations for the Convolution and Compression layers, a variety of functions, including Identity, ReLU and Sigmoid, are examined. Baseline model kNN-Content in Section 4.3 is used to assess the quality of the compressed genome scores. In more detail, 1128 genome scores of each movie are fed into the HCAE in order to extract its most fundamental features which are employed to calculate PCC $_{\text {genome }}$ similarity between movies in kNN-Content model using Equation (7) with $k=10$. Table 3 illustrates the performance of various activations in the case of compressing Tag Genome to $N=600$ features, where only RMSE are shown for brevity. Experiments with different values of $N$ provide the same results: a combination of (Identity, ReLU) provides the lowest error rate and is chosen for the Half Convolutional encoder thereafter.

Table 3. Performance of kNN-Content model with different activation functions of the Convolution and Compression layers in the HCAE.

\begin{tabular}{|c|c|c|c|}
\hline \multicolumn{2}{|c|}{ Activation Function } & \multirow{2}{*}{ RMSE } & \multirow{2}{*}{ Time [s] } \\
\hline Convolution Layer & Compression Layer & & \\
\hline Identity & Identity & 0.7856 & 295 \\
\hline Identity & $\underline{\operatorname{ReLU}}$ & $\underline{0.7608}$ & $\underline{297}$ \\
\hline Identity & Sigmoid & $\overline{0.7638}$ & $\overline{298}$ \\
\hline ReLU & Identity & 0.7854 & 296 \\
\hline ReLU & ReLU & 0.7739 & 297 \\
\hline ReLU & Sigmoid & 0.7641 & 300 \\
\hline Sigmoid & Identity & 0.7976 & 298 \\
\hline Sigmoid & ReLU & 0.7932 & 299 \\
\hline Sigmoid & Sigmoid & 0.7678 & 302 \\
\hline
\end{tabular}

To thoroughly evaluate the capability of the HCAE against other AE architectures, both versions of genome tags, 1128 original and 1044 combined ones using NLP [39], are chosen as their inputs. Specifically, a vanilla AE and its two popular variants are implemented as follows:

- $\quad$ AE: a 3-hidden layer AE that uses ReLU activation function at all hidden layers and Sigmoid activation function at the output layer; the number of units at hidden layers $\# 1$ and \#3 is fixed at 900;

- DAE: a 1-hidden layer DAE with noise_factor $=0.1$ that uses ReLU activation function at the hidden layer and Sigmoid activation function at the output layer; 
- VAE: a 4-hidden layer VAE is configured as follows: 900-unit hidden layers \#1 and \#4 use ReLU activation function; Compression layer constructed from Mean layer and Var layer use Linear activation function; Sigmoid activation function is used for output layer.

In our experiments, the proposed HCAE and its counterparts are deployed with different sizes of Compression layer to find the optimal configuration for each model. All models are trained until saturation using Adam optimization algorithm and the learning rate of 0.001 . Figure 4 shows that although all $\mathrm{AE}$ architectures achieve the highest quality with the Compression layer of 600 units, they perform differently for two types of inputs: whilst the classic $\mathrm{AE}$ and its two well-known variants do not work well with the original tags, the proposed architecture operates almost equivalently regardless of whether the inputs are preprocessed or not. Furthermore, the HCAE proves superior in extracting essential features over its competitors for both cases. This suggests that the HCAE has the potential of discovering the hidden relationships under the original genome tags without the need of analyzing their semantic meanings, which helps to ignore the NLP-based preprocess. In more general situations, it is expected that the HCAE still demonstrates its virtue with various kinds of input, not limited to data with meaningful labels. Hence, the 1128 original genome tags are selected for the remaining experiments hereafter.

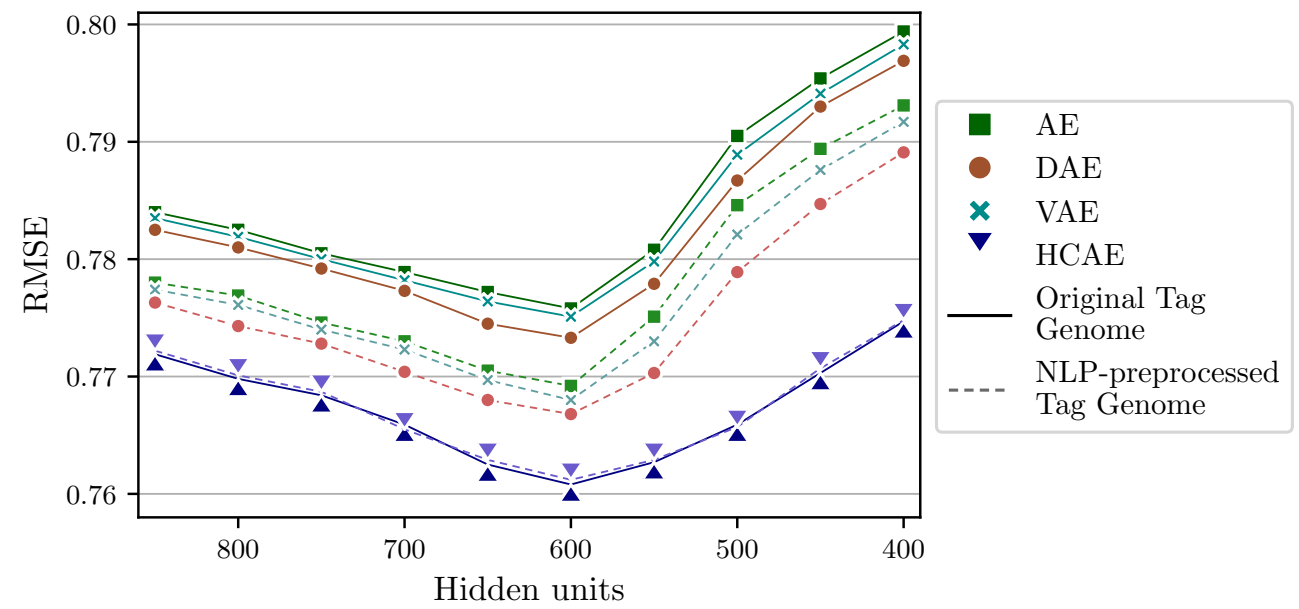

Figure 4. Error rates of $\mathbf{k N N}$-Content models using HCAE and the variants of AE with respect to the size of the Compression layer.

During the training process, we try shuffling the order of the genome scores as well as examining different values of the number of filters $(2,4,6,12,20)$ and the kernel sizes $(3,5,7,11,19,29)$ for the Convolution layer. Although the outputs of the Flatten and Compression layers vary constantly through the experiments, empirical results show that choosing the configuration of 4 filters and the kernel size of 5 provides the most accurate predictions in the final model but does not have a considerable improvement over other choices (the error rates fluctuate less than $0.06 \%$ ). This result helps to confirm our initial hypothesis that the Half Convolutional encoder could derive latent characteristics from the input data irrespective of the relationship and the positioning of the movie attributes.

For a more comprehensive evaluation, 600-feature vectors for movies generated from different types of $\mathrm{AE}$ are then applied into a number of baseline models: kNN-Content, FM $_{\text {genome }}, \mathrm{kNN}-$ Content-SVD, and kNN-Content-SVD++. It is noteworthy that in the cases of AE/DAE/VAE, feature vectors are compressed from the 1044 NLP-preprocessed genome tags for fair comparison because it provides better movie representation than using the original tags. In Table 4, the relative improvements of the HCAE-based models over their counterparts are displayed in the parentheses under the corresponding accuracy indicators. Here, $+/-$ signs indicate that the indicators of the proposed models are "greater" /"less" than the ones of their competitors, where the lower the RMSE or the 
higher the Precision/Recall the model gets, the better the performance is. This notation is also applied in the rest of this paper. Experimental results show that kNN-Content ${ }^{\mathrm{HCAE}}$ model gains the largest decreases in RMSE compared to $\mathrm{kNN}-$ Content ${ }^{\mathrm{AE}}$ model by $1.09 \%$ while also achieving at least $0.55 \%$ improvement in Precision/Recall. The other HCAEbased models also outperform their AE counterparts by at least $0.53 \%$ at the expense of operating time. Similar results can be seen when comparing HCAE- against DAE- and VAE-based models. A remarkable point here is that DAE performs better than AE and VAE in extracting fundamental features for movies. A potential reason for the dominance of DAE is its ability to process "noisy" genome scores calculated from user-contributed tags which possibly suffer from artificial errors. Even though the proposed HCAE still yield $0.58-0.78 \%$ lower RMSE and $0.43-0.73 \%$ higher Precision/Recall than DAE in all models.

Table 4. Performance comparison of the baseline models when utilizing 600-element movie feature vectors generated from AE variants and an HCAE.

\begin{tabular}{|c|c|c|c|c|c|c|c|}
\hline Model & $\begin{array}{c}\text { Auto } \\
\text { Encoder }\end{array}$ & RMSE & P@5 & P@10 & $\mathbf{R} @ 5$ & $\mathrm{R} @ 10$ & Time [s] \\
\hline \multirow{4}{*}{$\begin{array}{l}\text { kNN- } \\
\text { Content }\end{array}$} & $\mathrm{AE}$ & $\begin{array}{c}0.7692 \\
(-1.09 \%)\end{array}$ & $\begin{array}{c}0.8142 \\
(+0.68 \%)\end{array}$ & $\begin{array}{c}0.7906 \\
(+0.79 \%)\end{array}$ & $\begin{array}{c}0.4352 \\
(+0.55 \%)\end{array}$ & $\begin{array}{c}0.5587 \\
(+0.68 \%)\end{array}$ & 289 \\
\hline & VAE & $\begin{array}{c}0.7680 \\
(-0.94 \%)\end{array}$ & $\begin{array}{c}0.8137 \\
(+0.74 \%)\end{array}$ & $\begin{array}{c}0.7914 \\
(+0.69 \%)\end{array}$ & $\begin{array}{c}0.4358 \\
(+0.41 \%)\end{array}$ & $\begin{array}{c}0.5592 \\
(+0.59 \%)\end{array}$ & 316 \\
\hline & DAE & $\begin{array}{c}0.7668 \\
(-0.78 \%)\end{array}$ & $\begin{array}{c}0.8147 \\
(+0.62 \%)\end{array}$ & $\begin{array}{c}0.7925 \\
(+0.55 \%)\end{array}$ & $\begin{array}{c}0.4355 \\
(+0.48 \%)\end{array}$ & $\begin{array}{c}0.5588 \\
(+0.66 \%)\end{array}$ & 291 \\
\hline & HCAE & 0.7608 & $\underline{0.8198}$ & 0.7969 & $\underline{0.4376}$ & $\underline{0.5625}$ & 297 \\
\hline \multirow{4}{*}{$\mathrm{FM}_{\text {genome }}$} & $\mathrm{AE}$ & $\begin{array}{c}0.7702 \\
(-0.87 \%)\end{array}$ & $\begin{array}{c}0.8052 \\
(+0.62 \%)\end{array}$ & $\begin{array}{c}0.7836 \\
(+0.72 \%)\end{array}$ & $\begin{array}{c}0.4300 \\
(+0.78 \%)\end{array}$ & $\begin{array}{c}0.5571 \\
(+0.71 \%)\end{array}$ & 23,412 \\
\hline & VAE & $\begin{array}{c}0.7688 \\
(-0.69 \%) \\
\end{array}$ & $\begin{array}{c}0.8057 \\
(+0.56 \%) \\
\end{array}$ & $\begin{array}{c}0.7841 \\
(+0.66 \%) \\
\end{array}$ & $\begin{array}{c}0.4295 \\
(+0.90 \%) \\
\end{array}$ & $\begin{array}{c}0.5578 \\
(+0.59 \%) \\
\end{array}$ & 23,535 \\
\hline & DAE & $\begin{array}{c}0.7681 \\
(-0.60 \%)\end{array}$ & $\begin{array}{c}0.8063 \\
(+0.48 \%)\end{array}$ & $\begin{array}{c}0.7852 \\
(+0.52 \%)\end{array}$ & $\begin{array}{c}0.4310 \\
(+0.55 \%)\end{array}$ & $\begin{array}{c}0.5587 \\
(+0.43 \%)\end{array}$ & 23,447 \\
\hline & HCAE & $\underline{0.7635}$ & $\underline{0.8102}$ & $\underline{0.7893}$ & $\underline{0.4334}$ & $\underline{0.5611}$ & 23,503 \\
\hline \multirow{4}{*}{$\begin{array}{l}\text { kNN- } \\
\text { Content- } \\
\text { SVD }\end{array}$} & $\mathrm{AE}$ & $\begin{array}{c}0.7634 \\
(-0.98 \%)\end{array}$ & $\begin{array}{c}0.8162 \\
(+0.78 \%)\end{array}$ & $\begin{array}{c}0.7946 \\
(+0.76 \%)\end{array}$ & $\begin{array}{c}0.4356 \\
(+0.53 \%)\end{array}$ & $\begin{array}{c}0.5575 \\
(+0.78 \%)\end{array}$ & 596 \\
\hline & VAE & $\begin{array}{c}0.7623 \\
(-0.84 \%) \\
\end{array}$ & $\begin{array}{c}0.8166 \\
(+0.73 \%) \\
\end{array}$ & $\begin{array}{c}0.7941 \\
(+0.82 \%) \\
\end{array}$ & $\begin{array}{c}0.4349 \\
(+0.68 \%) \\
\end{array}$ & $\begin{array}{c}0.5580 \\
(+0.69 \%) \\
\end{array}$ & 653 \\
\hline & DAE & $\begin{array}{c}0.7612 \\
(-0.70 \%)\end{array}$ & $\begin{array}{c}0.8177 \\
(+0.60 \%)\end{array}$ & $\begin{array}{c}0.7954 \\
(+0.66 \%)\end{array}$ & $\begin{array}{c}0.4355 \\
(+0.55 \%)\end{array}$ & $\begin{array}{c}0.5594 \\
(+0.44 \%)\end{array}$ & 603 \\
\hline & HCAE & $\underline{0.7559}$ & $\underline{0.8226}$ & $\underline{0.8007}$ & $\underline{0.4379}$ & $\underline{0.5619}$ & 612 \\
\hline \multirow{4}{*}{$\begin{array}{l}\text { kNN- } \\
\text { Content- } \\
\text { SVD++ }\end{array}$} & $\mathrm{AE}$ & $\begin{array}{c}0.7584 \\
(-0.90 \%)\end{array}$ & $\begin{array}{c}0.8186 \\
(+0.78 \%)\end{array}$ & $\begin{array}{c}0.7959 \\
(+0.90 \%)\end{array}$ & $\begin{array}{c}0.4368 \\
(+0.66 \%)\end{array}$ & $\begin{array}{c}0.5609 \\
(+0.69 \%)\end{array}$ & 27,687 \\
\hline & VAE & $\begin{array}{c}0.7569 \\
(-0.70 \%) \\
\end{array}$ & $\begin{array}{c}0.8191 \\
(+0.72 \%) \\
\end{array}$ & $\begin{array}{c}0.7962 \\
(+0.86 \%) \\
\end{array}$ & $\begin{array}{c}0.4363 \\
(+0.77 \%) \\
\end{array}$ & $\begin{array}{c}0.5610 \\
(+0.67 \%) \\
\end{array}$ & 27,761 \\
\hline & DAE & $\begin{array}{c}0.7560 \\
(-0.58 \%)\end{array}$ & $\begin{array}{c}0.8198 \\
(+0.63 \%)\end{array}$ & $\begin{array}{c}0.7972 \\
(+0.73 \%) \\
\end{array}$ & $\begin{array}{c}0.4374 \\
(+0.52 \%) \\
\end{array}$ & $\begin{array}{c}0.5617 \\
(+0.55 \%)\end{array}$ & 27,698 \\
\hline & HCAE & $\underline{0.7516}$ & $\underline{0.8250}$ & $\underline{0.8031}$ & $\underline{0.4397}$ & $\underline{0.5648}$ & 27,737 \\
\hline
\end{tabular}

It is worth noting that the operating time of AE/DAE/VAE here does not take into account the step of NLP preprocessing which is totally eliminated when utilizing the proposed HCAE. In practice, the preprocessing stage introduced in [39] may cost a great 
amount of time if the number of tags increases sharply, which is highly likely to happen because they are freely generated by users. Therefore, the trade-off is totally acceptable.

\section{Novel Initialization Method Using Content-Based Information for Matrix Factorization Techniques}

As introduced in Section 2.2, matrix factorization is a well-known technique for discovering latent features. Although this technique performs well on extremely sparse data such as rating matrix, it lacks explainability: latent factors achieved after training a matrix factorization model are most likely uninterpretable. Furthermore, traditional matrix factorization methods randomly initialize user and item vectors during the training process that may lead to slow convergence. Especially, NMF is greatly affected by the initialization: if the initial values of $p_{u}$ and $q_{i}$ are not good, it is more likely that the training will be unstable, and even unable to converge [11,48]. Recent years have witnessed a large number of efforts to eliminate these problems. One of the highly promising approaches is to initialize the latent vectors with more meaningful values $[15,16]$.

In this paper, a new method of initialization in matrix factorization is proposed: instead of learning user and item features from randomly valued vectors, we attempt to integrate both content- and rating-based information into the initial vectors. Currently, one of the common ways to describe user preferences is using the interactions that a user assigned to the movies she watched. However, by using this method, rating data and movie attributes are treated independently, which may cause a huge waste of information. To tackle this issue, a new way of generally profiling a user that incorporates content-based data of all the movies she rated is introduced as follows.

$$
P_{u}=\frac{1}{|R(u)|} \sum_{i \in R(u)}\left(\tilde{r}_{u i} \times Q_{i}\right)
$$

where $\tilde{r}_{u i}$ denotes the rating user $u$ gave to movie $i$ which has been normalized to the range of $[0,1]$, and $Q_{i}$ is the feature vector of movie $i$ that reflects its content-related information. Recall that $R(u)$ denotes the set of all movies rated by user $u$. In such a manner, a user vector $P_{u}$ has the same dimension and range of values as a movie vector $Q_{i}$. More importantly, each user is characterized in an explainable way: elements with grearer values indicate that the user has a greater preference for the respective movie attributes and vice versa.

Finally, both the user and movie latent features of the matrix factorization model are initialized using the vectors $P_{u}$ 's and $Q_{i}$ 's described above. During the scope of this work, the Tag Genome is utilized to demonstrate the content of each movie. In order to comprehensively evaluate the new method of initialization, different representations of a movie based on these metadata are experimented: the 1128 original genome tags, the shortened 1044 ones using NLP [39], and the two 600-element feature vectors generated using the newly proposed HCAE and the DAE which yield the best results in the previous section. We refer to the proposed models with-genome suffix to indicate that the user and movie genome scores are integrated into the learning process where the number of latent factors $k$ must match the dimension of corresponding feature vectors. In our experiments, SVD-genome and NMF-genome models are trained with the learning rate of 0.005 and the regularization strength of 0.02 . The randomly initialized counterparts are trained 20 times using the same hyperparameters to select the most precise models for comparison. For clarity, we only include RMSE in Table 5. 
Table 5. Performance comparison between randomly initialized matrix factorization models and their custom initialized counterparts integrating user and movie feature vectors.

\begin{tabular}{|c|c|c|c|}
\hline Model & RMSE & Epochs & Time [s] \\
\hline $\operatorname{NMF}(k=1128)$ & 0.7993 & 305 & 32,115 \\
\hline $\begin{array}{c}\text { NMF-genome } \\
\text { (1128 original scores) }\end{array}$ & $\begin{array}{c}0.7797 \\
(-2.45 \%)\end{array}$ & $\begin{array}{c}200 \\
(-34.43 \%)\end{array}$ & $\begin{array}{c}21,220 \\
(-33.92 \%)\end{array}$ \\
\hline $\operatorname{NMF}(k=1044)$ & 0.7987 & 290 & 18,802 \\
\hline $\begin{array}{c}\text { NMF-genome } \\
\text { (1044 NLP-preprocessed scores) }\end{array}$ & $\begin{array}{c}0.7792 \\
(-2.44 \%)\end{array}$ & $\begin{array}{c}165 \\
(-43.10 \%)\end{array}$ & $\begin{array}{c}10,843 \\
(-42.33 \%)\end{array}$ \\
\hline $\operatorname{NMF}(k=600)$ & 0.7984 & 265 & 9,937 \\
\hline $\begin{array}{c}\text { NMF-genome } \\
\text { (600 scores of DAE) }\end{array}$ & $\begin{array}{c}0.7718 \\
(-3.33 \%)\end{array}$ & $\begin{array}{c}125 \\
(-52.83 \%)\end{array}$ & $\begin{array}{c}4695 \\
(-52.75 \%)\end{array}$ \\
\hline $\begin{array}{c}\text { NMF-genome } \\
\text { (600 scores of HCAE) }\end{array}$ & $\begin{array}{c}0.7688 \\
(-3.71 \%)\end{array}$ & $\begin{array}{c}97 \\
(-63.40 \%)\end{array}$ & $\begin{array}{c}3729 \\
(-62.47 \%)\end{array}$ \\
\hline $\operatorname{SVD}(k=1128)$ & 0.7930 & 230 & 11,201 \\
\hline $\begin{array}{c}\text { SVD-genome } \\
\text { (1128 original scores) }\end{array}$ & $\begin{array}{c}0.7582 \\
(-4.39 \%)\end{array}$ & $\begin{array}{c}55 \\
(-76.09 \%)\end{array}$ & $\begin{array}{c}2713 \\
(-75.78 \%)\end{array}$ \\
\hline $\operatorname{SVD}(k=1044)$ & 0.7927 & 150 & 5895 \\
\hline $\begin{array}{c}\text { SVD-genome } \\
\text { (1044 NLP-preprocessed scores) }\end{array}$ & $\begin{array}{c}0.7547 \\
(-4.79 \%)\end{array}$ & $\begin{array}{c}45 \\
(-70.00 \%) \\
\end{array}$ & $\begin{array}{c}1787 \\
(-69.69 \%) \\
\end{array}$ \\
\hline $\operatorname{SVD}(k=600)$ & 0.7925 & 140 & 3018 \\
\hline $\begin{array}{l}\text { SVD-genome } \\
\text { (600 scores of DAE) }\end{array}$ & $\begin{array}{c}0.7536 \\
(-4.91 \%)\end{array}$ & $\begin{array}{c}35 \\
(-75.00 \%)\end{array}$ & $\begin{array}{c}905 \\
(-70.01 \%)\end{array}$ \\
\hline $\begin{array}{c}\text { SVD-genome } \\
(600 \text { scores of HCAE) }\end{array}$ & $\begin{array}{c}0.7472 \\
(-5.72 \%)\end{array}$ & $\begin{array}{c}30 \\
(-78.57 \%)\end{array}$ & $\begin{array}{c}814 \\
(-73.03 \%)\end{array}$ \\
\hline
\end{tabular}

As displayed in Table 5, incorporating rating data and movie attributes into the initialization significantly boosts SVD-genome and NMF-genome models over their original versions. In detail, the proposed models show an exceptionally faster convergence rate against the competitors in all experiments: the training time measured by the number of epochs is reduced to at least $34.43 \%$, especially up to $63.40 \%$ and $78.57 \%$ with the models utilizing HCAE-generated feature vectors. At the same time, SVD-genome and NMFgenome models totally outperform their corresponding counterparts from $2.44 \%$ to $5.72 \%$ in RMSE. Once again, it can be seen that initializing latent vectors with 600 scores produced by an HCAE (highlighted rows) provides the lowest error rate when compared with other representations, which further proves the superiority of the HCAE against AE variants stated in the previous section. These remarkable improvements prove that learning the user and item latent features from appropriate initial vectors could greatly benefit matrix factorization models: it not only helps the training process to converge faster but also enhances the accuracy. In real-life applications, the word "appropriate" here implies that we could take advantage of all accessible knowledge including user preferences and available metadata, not limited to user-contributed genome tags in the above experiments, in order to characterize user and item features as precisely as possible. This empirical result suggests that the more meaningful values of initial latent vectors, the more likely it is to achieve better matrix factorization-based RSs in terms of precision and computational complexity.

Another advantage of the newly proposed method is that if adopting the original 1128 genome tags or the shortened 1044 ones preprocessed by NLP for initialization, movie latent vectors generated after training have much better interpretability than the ones from traditional matrix factorization models. As demonstrated in Figure 5, the most relevant 
tags of Titanic (1997) deduced from the learned movie features are highly reasonable for both versions of the genome tags. For user latent vectors, there are clear differences before and after training (user with id $=1$ for example). However, it is reasonable to expect that learned user vectors could provide a better understanding of user preferences and can be employed for further analysis owing to their significant contribution towards generating RSs with higher accuracy.

The most suitable tags of the movie Titanic (1997)

\begin{tabular}{|c|c|c|c|}
\hline \multicolumn{2}{|c|}{ Original 1,128 Tag Genome } & \multicolumn{2}{|c|}{ Item-factor vector of SVD-genome 1,128 factors } \\
\hline 1. love story & 2.big budget & 1.sacrifice & 2.ocean \\
\hline 3.oscar-best picture & 4.romantic & 3.tragedy & 4. based on true story \\
\hline 5. special effects & 6.oscar-best directing & 5. disaster & 6. tear jerker \\
\hline 7.girlie movie & 8. romance & 7.oscar-best editing & 8. natural disaster \\
\hline 9. catastrophe & 10. natural disaster & 9. chick flick & 10.1ong \\
\hline 11. love & 12. chick flick & 11.epic & 12. historical \\
\hline 13. oscar & ar winner & 13. bittersweet & 14. love \\
\hline $15 . d$ & 16. & 15. special effects & 16. nudity (tople \\
\hline 17. historical & d on true & 17. survivalpicture & 18. oscar-best picture \\
\hline 19. sentimental & 20.te & 19. romantic & 20. big budget \\
\hline \multicolumn{2}{|c|}{ NLP-preprocesed 1,044 Tag Genome } & \multicolumn{2}{|c|}{ Item-factor vector of SVD-genome 1,044 factors } \\
\hline 1.big budget & 2.romar & 1.sacrifice & 2.ocean \\
\hline 3.love & 4.special effects & 3.tragedy & 4.girlie movie \\
\hline 5. oscar winner & 6.girlie movie & 5. tear jerker & 6.chick flick \\
\hline 7. catastrophe & 8. natural disaster & 7. natural disaster & 8.oscar winner \\
\hline 9. chick flick & 10. destiny & 9. bittersweet & 10.long \\
\hline 11. sentimental & 12. tear jerker & 11. based on a true stor & ry 12 .history \\
\hline 13. emotional & 14.epic & 13. epic & 14. nudity (topless) \\
\hline 15. touching & 16. history & 15. love & 16. survival \\
\hline 17. sacrifice & 18. bittersweet & 17. special & 18. romance \\
\hline 19.pg-13 & 20. courage & 19. period piece & 20. emotional \\
\hline
\end{tabular}

The most suitable tags of the user with $i d=1$

\begin{tabular}{|c|c|c|c|}
\hline \multicolumn{2}{|c|}{ Original 1,128 Tag Genome } & \multicolumn{2}{|c|}{ User-factor vector of SVD-genome 1,128 factors } \\
\hline 1.original & 2.great ending & 1. special effects & 2. big budget \\
\hline 3. storytelling & 4.imdb top 250 & 3. dark fantasy & 4. fantasy \\
\hline 5. story & 6. great & 5. cult film & 6. based on a comic \\
\hline 7. dialogue & 8. great movie & 7. book was better & 8. horror \\
\hline 9. good & 10. mentor & 9. superheroes & 10. story \\
\hline 11. good soundtrack & 12. cult classic & 11. mythology & 12. adapted from:comic \\
\hline 13. great acting & 14.visual & 13. super hero & 14. adaptation \\
\hline 15. visually appealing & 16. suspense & 15. modern fantasy & 16. awesome soundtrack \\
\hline 17. masterpiece & 18.interesting & 17. dynamic cgi action & 18. sci fi \\
\hline 19.classic & 20. adapted from:book & 19. science fiction & 20. super-hero \\
\hline
\end{tabular}

\begin{tabular}{|c|c|c|c|}
\hline \multicolumn{2}{|c|}{ NLP-preprocesed 1,044 Tag Genome } & \multicolumn{2}{|c|}{ User-factor vector of SVD-genome 1,044 factors } \\
\hline 1.great ending & 2.original plot & 1. special effects & 2. big budget \\
\hline 3. storytelling & 4.imdb top 250 & 3.story & 4. science fiction \\
\hline 5. story & 6.mentor & 5. super hero & 6. dark fantasy \\
\hline 7. cult classic & 8.masterpiece & 7. predictable & 8. adaptation \\
\hline 9.classic & 10. intense & 9. dynamic cgi action & 10. my thology \\
\hline 11.oscar winner & 12. atmospheric & 11. fantasy & 12. book was better \\
\hline 13. special effects & 14.clever & 13. shallow & 14.magic \\
\hline 15. cinematography & 16. adaptation & 15. alter ego & 16. modern fantasy \\
\hline 17. runaway & 18. adventure & 17.alternate reality & 18. dreamlike \\
\hline 19. violence & 20. chase & 19. horror & 20. first contact \\
\hline
\end{tabular}

Figure 5. The 20 most relevant tags of the movie Titanic (1997) and the user with $i d=1$ based on the original Tag Genome and the feature vectors after training SVD-genome.

The two most accurate models, NMF-genome and SVD-genome utilizing $600 \mathrm{HCAE}-$ generated scores, are chosen to compare with the baselines listed in Section 4.3. In this final experiment, the baselines are implemented using different libraries and frameworks in order to pick up the model with the lowest error rate, not considering the overall time to finish the training and predicting process. In other words, we only evaluate the models in terms of accuracy. Therefore, only accuracy indicators are displayed in Table 6 for fair comparison. Experimental results show that the proposed models greatly surpass the competitors. Specifically, the highlighted winning model, SVD-genome, has gained an improvement from approximately $0.59 \%$ to $7.13 \%$ over the baselines at both rating prediction and ranking tasks. These results are encouraging, and it is highly likely that a much lower error rate could be attained if the proposed initialization method is applied to SVD++ and its current state-of-the-art variants such as timeSVD++ [8] and 
flippedTimeSVD++ [49]. However, due to our limited hardware resources, this experiment cannot be carried out: using a large number of latent factors causes the training process to take an enormous amount of time to finish. In real-life applications, this huge trade-off between accuracy and computational complexity is usually not worth the effort.

Table 6. Performance comparison between the proposed models utilizing 600 HCAE-generated feature vectors and the baseline models.

\begin{tabular}{cccccc}
\hline Model & RMSE & P@5 & P@10 & R@5 & R@10 \\
\hline \multirow{2}{*}{ ii-CF } & 0.8046 & 0.7967 & 0.7721 & 0.4261 & 0.5541 \\
& $(-7.13 \%)$ & $(+4.06 \%)$ & $(+4.45 \%)$ & $(+3.79 \%)$ & $(+3.20 \%)$ \\
I-AutoRec & 0.7808 & 0.7778 & 0.7559 & 0.3972 & 0.5228 \\
& $(-4.30 \%)$ & $(+6.33 \%)$ & $(+6.46 \%)$ & $(+10.32 \%)$ & $(+8.67 \%)$ \\
NMF & 0.7981 & 0.7951 & 0.7743 & 0.4296 & 0.5583 \\
& $(-6.38 \%)$ & $(+4.25 \%)$ & $(+4.18 \%)$ & $(+3.00 \%)$ & $(+2.46 \%)$ \\
SVD & 0.7922 & 0.8005 & 0.7786 & 0.4322 & 0.5628 \\
& $(-5.68 \%)$ & $(+3.60 \%)$ & $(+3.65 \%)$ & $(+2.42 \%)$ & $(+1.68 \%)$ \\
SVD++ & 0.7894 & 0.8030 & 0.7817 & 0.4339 & 0.5639 \\
& $(-5.35 \%)$ & $(+3.30 \%)$ & $(+3.27 \%)$ & $(+2.03 \%)$ & $(+1.48 \%)$ \\
\hline \multirow{2}{*}{ kNN-Content ${ }^{H C A E}-S V D$} & 0.7559 & 0.8226 & 0.8007 & 0.4379 & 0.5619 \\
& $(-1.15 \%)$ & $(+0.94 \%)$ & $(+0.92 \%)$ & $(+1.13 \%)$ & $(+1.83 \%)$ \\
kNN-Content HCAE_SVD++ & 0.7516 & 0.8250 & 0.8031 & 0.4397 & 0.5648 \\
& $(-0.59 \%)$ & $(+0.65 \%)$ & $(+0.62 \%)$ & $(+0.72 \%)$ & $(+1.33 \%)$ \\
\hline \multirow{2}{*}{ NMF-genome } & 0.7688 & 0.8174 & 0.7941 & 0.4389 & 0.5689 \\
SVD-genome & $(-2.81 \%)$ & $(+1.57 \%)$ & $(+1.73 \%)$ & $(+0.90 \%)$ & $(+0.61 \%)$ \\
& $\underline{\mathbf{0 . 7 4 7 2}}$ & $\underline{\mathbf{0 . 8 3 0 4}}$ & $\underline{\mathbf{0 . 8 0 8 1}}$ & $\underline{\mathbf{0 . 4 4 2 9}}$ & $\mathbf{0 . 5 7 2 4}$ \\
\hline
\end{tabular}

\section{Conclusions}

In this work, a novel architecture of autoencoder named HCAE is first proposed in order to discover essential information from the original Tag Genome of each movie. By integrating a 1D-CNN into the encoder part, an HCAE proves its capability in feature extraction compared to a vanilla $\mathrm{AE}$ and its variants, especially without the need for the time-consuming data preprocessing stage. Secondly, a new method of profiling users is introduced using the HCAE-generated movie feature vectors and rating information. The new representations of users and movies are then utilized as the initial latent vectors during the training stage of common matrix factorization techniques. In addition to learning movie feature vectors with better explainability, experimental results demonstrate that the custom-initialized SVD/NMF models not only converge much faster in the training but also outperform the randomly initialized counterparts in both rating prediction and ranking tasks. It is thus expected that matrix factorization-based RSs could be greatly improved via generating robust user and item profiles from all available information for initialization.

The proposed HCAE has enabled several potential ideas on applying state-of-the-art feature extractors such as a deep CNN or a transformer-based AE to generate robust hidden feature vectors. Another potential direction is to adopt convolutional or transformer-based models not only as an AE, but as an end-to-end recommendation model.

Author Contributions: Conceptualization, T.N.D., N.N.D. and T.G.D.; methodology, M.H.T.; software, Q.H.D.; validation, T.N.D., D.M.N. and M.H.T.; formal analysis, T.N.D.; investigation, T.N.D., N.N.D. and T.G.D.; resources, T.N.D.; data curation, T.N.D. and N.N.D.; writing-original draft preparation, N.N.D. and T.G.D.; writing-review and editing, T.N.D. and Q.H.D.; visualization, T.G.D.; funding acquisition, D.M.N. All authors have read and agreed to the published version of the manuscript.

Funding: This research was funded by the Project of Hanoi University of Science and Technology under Grant no. T2020-PC-024. 


\section{Institutional Review Board Statement: Not applicable \\ Informed Consent Statement: Not applicable}

Data Availability Statement: Not applicable

Conflicts of Interest: The authors declare no conflict of interest. The funders had no role in the design of the study; in the collection, analyses, or interpretation of data; in the writing of the manuscript, or in the decision to publish the results.

\section{References}

1. Adomavicius, G.; Tuzhilin, A. Toward the next generation of recommender systems: A survey of the state-of-the-art and possible extensions. IEEE Trans. Knowl. Data Eng. 2005, 17, 734-749. [CrossRef]

2. Lops, P.; De Gemmis, M.; Semeraro, G. Content-based recommender systems: State of the art and trends. In Recommender Systems Handbook; Springer: Cham, Switzerland, 2011; pp. 73-105

3. Narducci, F.; Basile, P.; Musto, C.; Lops, P.; Caputo, A.; de Gemmis, M.; Iaquinta, L.; Semeraro, G. Concept-based item representations for a cross-lingual content-based recommendation process. Inf. Sci. 2016, 374, 15-31. [CrossRef]

4. Su, X.; Khoshgoftaar, T.M. A Survey of Collaborative Filtering Techniques. Available online: https://downloads.hindawi.com/ archive/2009/421425.pdf (accessed on 2 November 2021).

5. Ricci, F.; Rokach, L.; Shapira, B. Recommender systems: Introduction and challenges. In Recommender Systems Handbook; Springer: Cham, Switzerland 2015; pp. 1-34.

6. Funk, S. Netflix Update: Try This at Home, 2006. Available online: https://sifter.org/simon/journal/20061211.html (accessed on 2 November 2021).

7. Koren, Y. Factorization meets the neighborhood: A multifaceted collaborative filtering model. In Proceedings of the 14th ACM SIGKDD International Conference on Knowledge Discovery and Data Mining, Las Vegas, NV, USA, 24-27 August 2008; pp. 426-434.

8. Koren, Y. Collaborative filtering with temporal dynamics. In Proceedings of the 15th ACM SIGKDD International Conference on Knowledge Discovery and Data Mining, Paris, France, 28 June-1 July 2009; pp. 447-456.

9. Wild, S.; Curry, J.; Dougherty, A. Improving non-negative matrix factorizations through structured initialization. Pattern Recognit. 2004, 37, 2217-2232. [CrossRef]

10. Boutsidis, C.; Gallopoulos, E. SVD based initialization: A head start for nonnegative matrix factorization. Pattern Recognit. 2008, 41, 1350-1362. [CrossRef]

11. Albright, R.; Cox, J.; Duling, D.; Langville, A.N.; Meyer, C. Algorithms, Initializations, and Convergence for the Nonnegative Matrix Factorization. Available online: https:/ / www.ime.usp.br/ jmstern/wp-content/uploads/2020/04/Albright1.pdf (accessed on 2 November 2021).

12. Agarwal, D.; Chen, B.C. Regression-based latent factor models. In Proceedings of the 15th ACM SIGKDD International Conference on Knowledge Discovery and Data Mining, Paris France, 28 June-1 July 2009; pp. 19-28.

13. Barragáns-Martínez, A.B.; Costa-Montenegro, E.; Burguillo, J.C.; Rey-López, M.; Mikic-Fonte, F.A.; Peleteiro, A. A hybrid contentbased and item-based collaborative filtering approach to recommend TV programs enhanced with singular value decomposition. Inf. Sci. 2010, 180, 4290-4311. [CrossRef]

14. Rendle, S. Factorization machines. In Proceedings of the 2010 IEEE International Conference on Data Mining, Sydney, Australia, 13-17 December 2010; pp. 995-1000.

15. Hidasi, B.; Tikk, D. Initializing Matrix Factorization Methods on Implicit Feedback Databases. J. UCS 2013, 19, $1834-1853$.

16. Zhao, J.; Geng, X.; Zhou, J.; Sun, Q.; Xiao, Y.; Zhang, Z.; Fu, Z. Attribute mapping and autoencoder neural network based matrix factorization initialization for recommendation systems. Knowl. Based Syst. 2019, 166, 132-139. [CrossRef]

17. He, X.; Liao, L.; Zhang, H.; Nie, L.; Hu, X.; Chua, T.S. Neural Collaborative Filtering. In Proceedings of the 26th International Conference on World Wide Web, International World Wide Web Conferences Steering Committee, Republic and Canton of Geneva, Switzerland, 3-7 April 2017; pp. 173-182.

18. Martins, G.B.; Papa, J.P.; Adeli, H. Deep learning techniques for recommender systems based on collaborative filtering. Expert Syst. 2020, 37, e12647. [CrossRef]

19. LeCun, Y.; Bengio, Y. Convolutional Networks for Images, Speech, and Time Series. Available online: www.iro.umontreal.ca/ lisa/pointeurs/handbook-convo.pdf (accessed on 2 November 2021).

20. Abdul, A.; Chen, J.; Liao, H.Y.; Chang, S.H. An emotion-aware personalized music recommendation system using a convolutional neural networks approach. Appl. Sci. 2018, 8, 1103. [CrossRef]

21. Kim, D.; Park, C.; Oh, J.; Lee, S.; Yu, H. Convolutional matrix factorization for document context-aware recommendation. In Proceedings of the 10th ACM Conference on Recommender Systems, Boston, MA, USA, 15-19 September 2016; pp. 233-240.

22. Harper, F.M.; Konstan, J.A. The movielens datasets: History and context. ACM Trans. Interact. Intell. Syst. (TIIS) 2016, 5, 19. [CrossRef] 
23. Herlocker, J.L.; Konstan, J.A.; Borchers, A.; Riedl, J. An algorithmic framework for performing collaborative filtering. In Proceedings of the 22nd Annual International ACM SIGIR Conference on Research and Development in Information Retrieval, SIGIR 1999, Berkeley, CA, USA, 15-19 August 1999.

24. Sarwar, B.M.; Karypis, G.; Konstan, J.A.; Riedl, J. Item-Based Collaborative Filtering Recommendation Algorithms. Available online: https://dl.acm.org/doi/pdf/10.1145/371920.372071?casa_token=r5ThY9p5rlIAAAAA:RWJZHgJ14YQsoHgKGGJvFWuQe8 vU9-deU51KUxCQaxykLNW1nmAvqcX11_SVKtwPSJYhTaXV47ujrA (accessed on 2 November 2021).

25. Koren, Y. Factor in the neighbors: Scalable and accurate collaborative filtering. ACM Trans. Knowl. Discov. Data (TKDD) $2010,4,1$. [CrossRef]

26. Duong, T.N.; Than, V.D.; Tran, T.H.; Dang, Q.H.; Nguyen, D.M.; Pham, H.M. An Effective Similarity Measure for Neighborhoodbased Collaborative Filtering. In Proceedings of the 2018 5th NAFOSTED Conference on Information and Computer Science (NICS), Ho Chi Minh City, Vietnam, 23-24 November 2018;pp. 250-254.

27. Zhang, S.; Wang, W.; Ford, J.; Makedon, F. Learning from incomplete ratings using non-negative matrix factorization. In Proceedings of the 2006 SIAM International Conference on Data Mining, Bethesda, MD, USA, 20-22 April 2006 ; pp. 549-553.

28. Gemulla, R.; Nijkamp, E.; Haas, P.J.; Sismanis, Y. Large-scale matrix factorization with distributed stochastic gradient descent. In Proceedings of the 17th ACM SIGKDD International Conference on Knowledge Discovery and Data Mining, San Diego, CA, USA, 21-24 August 2011; pp. 69-77.

29. Bao, Y.; Fang, H.; Zhang, J. Topicmf: Simultaneously exploiting ratings and reviews for recommendation. In Proceedings of the AAAI Conference on Artificial Intelligence, 2014, Québec City, QC, Canada, 27-31 July 2014.

30. Zhang, Y.; Chen, X. Explainable recommendation: A survey and new perspectives. arXiv 2018, arXiv:1804.11192.

31. Hinton, G.E.; Salakhutdinov, R.R. Reducing the dimensionality of data with neural networks. Science 2006, $313,504-507$. [CrossRef] [PubMed]

32. Vincent, P.; Larochelle, H.; Lajoie, I.; Bengio, Y.; Manzagol, P.A.; Bottou, L. Stacked Denoising Autoencoders: Learning Useful Representations in a Deep Network with a Local Denoising Criterion. Available online: https://www.jmlr.org/papers/volume1 1/vincent10a/vincent10a.pdf?source=post_page (accessed on 2 November 2021).

33. Kingma, D.P.; Welling, M. Auto-Encoding Variational Bayes. arXiv 2014, arXiv:1312.6114.

34. Wang, H.; Wang, N.; Yeung, D.Y. Collaborative deep learning for recommender systems. In Proceedings of the 21th ACM SIGKDD International Conference on knowledge Discovery and Data Mining, Sydney, NSW, Australia, 10-13 August 2015; pp $1235-1244$

35. Sedhain, S.; Menon, A.K.; Sanner, S.; Xie, L. Autorec: Autoencoders meet collaborative filtering. In Proceedings of the 24th International Conference on World Wide Web, Florence, Italy, 8-22 May 2015; pp. 111-112.

36. Jhamb, Y.; Ebesu, T.; Fang, Y. Attentive contextual denoising autoencoder for recommendation. In Proceedings of the 2018 ACM SIGIR International Conference on Theory of Information Retrieval, Tianjin, China, 14-17 September 2018; pp. 27-34.

37. Wang, R.; Jiang, Y.; Lou, J. TDR: Two-stage deep recommendation model based on mSDA and DNN. Expert Syst. Appl. 2020, 145, 113116. [CrossRef]

38. Duong, T.N.; Than, V.D.; Vuong, T.A.; Tran, T.H.; Dang, Q.H.; Nguyen, D.M.; Pham, H.M. A Novel Hybrid Recommendation System Integrating Content-Based and Rating Information. In Proceedings of the International Conference on Network-Based Information Systems 2019, Oita, Japan, 5-7 September 2019; pp. 325-337.

39. Duong, T.N.; Vuong, T.A.; Nguyen, D.M.; Dang, Q.H. Utilizing an Autoencoder-Generated Item Representation in Hybrid Recommendation System. IEEE Access 2020, 8, 75094-75104. [CrossRef]

40. Bennett, J.; Lanning, S. The netflix prize. In Proceedings of the KDD Cup and Workshop, New York, NY, USA, 12 August 2007; Volume 2007, p. 35

41. Takahashi, N.; Katayama, J.; Takeuchi, J. A generalized sufficient condition for global convergence of modified multiplicative updates for NMF. In Proceedings of the 2014 International Symposium on Nonlinear Theory and Its Applications, Luzern, Switzerland, 14-18 September 2014; pp. 44-47.

42. Lam, S.K.; Pitrou, A.; Seibert, S. Numba: A llvm-based python jit compiler. In Proceedings of the Second Workshop on the LLVM Compiler Infrastructure in HPC, Austin, TX, USA, 15 November 2015; pp. 1-6.

43. Ivan, C. Convolutional Neural Networks on Randomized Data. In Proceedings of the CVPR Workshops, Long Beach, CA, USA, 16-20 June 2019; pp. 1-8.

44. Kiranyaz, S.; Avci, O.; Abdeljaber, O.; Ince, T.; Gabbouj, M.; Inman, D.J. 1D convolutional neural networks and applications: A survey. Mech. Syst. Signal Process. 2021, 151, 107398. [CrossRef]

45. Zhang, W.; Peng, G.; Li, C. Bearings fault diagnosis based on convolutional neural networks with 2-D representation of vibration signals as input. In Proceedings of the MATEC Web of Conferences, EDP Sciences, Sibiu, Romania, 7-9 June 2017; Volume 95, p. 13001.

46. Hoang, D.T.; Kang, H.J. Convolutional neural network based bearing fault diagnosis. In Proceedings of the International Conference on Intelligent Computing, Madurai, India, 15-16 June 2017; pp. 105-111.

47. Kingma, D.P.; Ba, J. Adam: A Method for Stochastic Optimization. arXiv 2017, arXiv:1412.6980.

48. Wild, S.; Wild, W.S.; Curry, J.; Dougherty, A.; Betterton, M. Seeding Non-Negative Matrix Factorizations with the Spherical K-Means Clustering. Ph.D. Thesis, University of Colorado, Boulder, CO, USA, 2003.

49. Rendle, S. Scaling factorization machines to relational data. Proc. VLDB Endow. 2013, 6, 337-348. [CrossRef] 